\title{
Clock-dependent chromatin topology modulates circadian transcription and behavior
}

\author{
Jérôme Mermet, ${ }^{1,4}$ Jake Yeung, ${ }^{1,4}$ Clémence Hurni, ${ }^{1}$ Daniel Mauvoisin ${ }^{1}$ Kyle Gustafson, ${ }^{1}$ \\ Céline Jouffe, ${ }^{2}$ Damien Nicolas, ${ }^{1}$ Yann Emmenegger, ${ }^{3}$ Cédric Gobet, ${ }^{1,2}$ Paul Franken, ${ }^{3}$ \\ Frédéric Gachon, ${ }^{1,2}$ and Félix Naef ${ }^{1}$ \\ ${ }^{1}$ School of Life Sciences, Ecole Polytechnique Fédérale de Lausanne (EPFL), CH-1015 Lausanne, Switzerland; ${ }^{2}$ Nestle Institute of \\ Health Sciences, CH-1015 Lausanne, Switzerland; ${ }^{3}$ Center for Integrative Genomics, University of Lausanne, CH-1015 Lausanne, \\ Switzerland
}

The circadian clock in animals orchestrates widespread oscillatory gene expression programs, which underlie 24-h rhythms in behavior and physiology. Several studies have shown the possible roles of transcription factors and chromatin marks in controlling cyclic gene expression. However, how daily active enhancers modulate rhythmic gene transcription in mammalian tissues is not known. Using circular chromosome conformation capture (4C) combined with sequencing (4C-seq), we discovered oscillatory promoter-enhancer interactions along the 24-h cycle in the mouse liver and kidney. Rhythms in chromatin interactions were abolished in arrhythmic Bmal1 knockout mice. Deleting a contacted intronic enhancer element in the Cryptochrome 1(Cry1) gene was sufficient to compromise the rhythmic chromatin contacts in tissues. Moreover, the deletion reduced the daily dynamics of Cry1 transcriptional burst frequency and, remarkably, shortened the circadian period of locomotor activity rhythms. Our results establish oscillating and clock-controlled promoter-enhancer looping as a regulatory layer underlying circadian transcription and behavior.

[Keywords: circadian rhythms; chromatin topology; promoter-enhancer loops; DNA regulatory elements; transcriptional bursting]

Supplemental material is available for this article.

Received January 26, 2018; revised version accepted March 2, 2018.

The circadian clock, encoded in a core genetic network, governs rhythms in behavior and physiology (Schibler et al. 2015), such as nocturnal activity in mice and oscillations in carbohydrate and lipid metabolism in the liver (Bass and Lazar 2016). This clock also orchestrates the daily rhythmic synthesis of thousands of transcripts by impinging on multiple gene regulatory layers (Zhang et al. 2014). These rhythmic transcripts often coincide with rhythms in chromatin modifications, DNA accessibility, enhancer activity, and transcription factor (TF) binding at promoter-proximal and promoter-distal regions (Mermet et al. 2017; Takahashi 2017), suggesting that chromatin interactions play a role in regulating circadian gene expression.

Chromatin architecture in the nucleus is organized over multiple scales (Dekker et al. 2013). At the fine scale, this organization involves the interactions between gene pro-

\footnotetext{
${ }^{4}$ These authors contributed equally to this work. Corresponding author: felix.naef@epfl.ch

Article published online ahead of print. Article and publication date are online at http://www.genesdev.org/cgi/doi/10.1101/gad.312397.118. Freely available online through the Genes \& Development Open Access option.
}

moters and enhancer DNA elements through promoterenhancer looping (Fulco et al. 2016). The remodeling of such DNA contacts and the accompanying dynamics of transcriptional responses have been investigated in the context of signal-dependent gene induction, cell differentiation, and developmental transitions (Palstra et al. 2003; Ghavi-Helm et al. 2014; Kuznetsova et al. 2015). However, little is known about the dynamics of DNA looping along the recurring daily $24-\mathrm{h}$ cycle and the consequences on clock-dependent gene expression in animals.

Cell culture models investigating genes of interest have suggested that nuclear compartmentalization modulates cyclic gene expression (Zhao et al. 2015) and that oscillatory contacts between gene promoters and genomic regions on trans chromosomes accompany rhythmic mRNA expression (Aguilar-Arnal et al. 2013). Recently, we described tissue-specific chromatin interactions selectively associated with rhythmically expressed clock output transcripts (Yeung et al. 2018), but, in general, the circadian dynamics of DNA interactions, including their

(C) 2018 Mermet et al. This article, published in Genes \& Development, is available under a Creative Commons License (Attribution 4.0 International), as described at http://creativecommons.org/licenses/by/4.0/. 
regulation of core clock function and control of circadian gene expression, remain an open question. Indeed, rhythmic transcription could be regulated over an established static promoter-enhancer network (Ghavi-Helm et al. 2014; Xu et al. 2016), or, conversely, the clock could drive dynamic promoter-enhancer looping for high-amplitude daily oscillations in transcription.

Here we monitored promoter-enhancer contacts of a core clock and metabolic clock output gene across time and genotypes in mouse tissues and discovered that contact frequencies oscillated along the 24-h cycle. In arrhythmic Bmal1 knockout animals, these oscillations were abolished. Deletion in mice of an enhancer that was rhythmically recruited to the Cryptochrome 1 (Cry1) promoter led to a short period phenotype in locomotor activity. Moreover, this deletion compromised rhythmic chromatin topology in the liver and led to reduced peak Cry1 mRNA expression levels. Finally, single-molecule RNA fluorescent in situ hybridization (smRNA-FISH) showed that the abolished rhythmic chromatin contact reduced the daily dynamics of Cry1 transcriptional burst frequency.

\section{Results}

\section{Rhythmic local chromatin interactions in mouse livers}

We focused on two genes representing key temporally regulated hepatic functions: a gene essential for the core circadian oscillator, Cry1 (Griffin et al. 1999; van der Horst et al. 1999|, and a liver-specific clock-controlled gene, Glycogen Synthase 2 (Gys2) (Doi et al. 2010), which encodes the rate-limiting enzyme in hepatic glycogen synthesis (Irimia et al. 2010). These transcripts are rhythmically expressed in the liver at opposite times of day, Cry1 peaking during the night at Zeitgeber time 20 (ZT20) and Gys2 peaking during the day at ZT08 (with ZT0 corresponding to lights on and ZT12 corresponding to lights off) (Supplemental Fig. S1A). Using circular chromosome conformation capture (4C) combined with sequencing (4C-seq) (Gheldof et al. 2012), we estimated the interaction frequencies of DNA bait fragments placed near the transcription start sites (TSSs) of Cry1 and Gys2 versus the entire genome in livers of wild-type mice collected at ZT08 and ZT20 ( $n=4$ per time point). 4C-seq signals around the Cry1 and Gys2 TSSs decayed to background levels following a power law (Supplemental Fig. S1B,C; Supplemental Table S1; Sanborn et al. 2015) and did not exceed background on trans chromosomes (Supplemental Fig. S1D,E; Supplemental Table S1). The high proportion of chromatin interactions within the first $2 \mathrm{Mb}$ surrounding the baits on the cis chromosome (Cry1 TSS: $41 \%$ of total cis contacts at ZT08 and $46 \%$ at ZT20; Gys2 TSS: $54 \%$ at ZT08 and 57\% at ZT20) indicated that Cry1 and Gys2 regulatory contacts were contained within this signal-rich region (Sanyal et al. 2012). To compare 4C-seq profiles across conditions, we normalized the data and applied locally weighted multilinear regression (LWMR), which uses a Gaussian window $(\sigma=2500 \mathrm{~kb}$ ) centered on each fragment for local smoothing (Materials and Methods). For Cry1, the 4C-seq profiles after LWMR were similar between ZT08 and ZT20 except in a region downstream from the Cry1 promoter, where the contact frequency was increased at ZT20 (Fig. 1A). While the

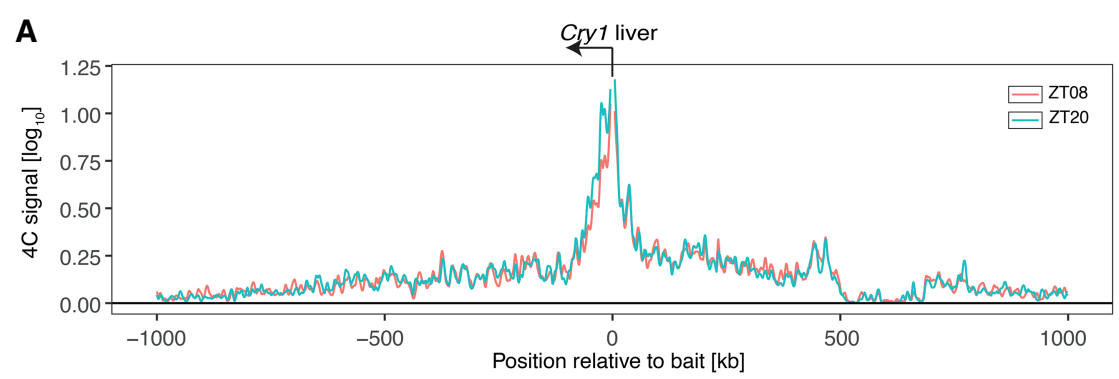

B

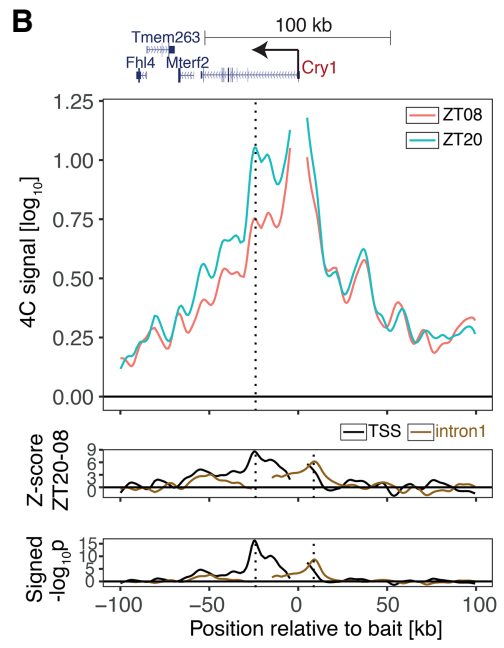

Figure 1. Rhythmic chromatin interactions in mouse livers. (A) 4C-seq data (LWMR summarizes $n=4$ animals per group) in a $2-\mathrm{Mb}$ genomic region surrounding Cry 1 at ZT08 and ZT20. (B) 4C-seq signals in a 200-kb genomic region surrounding Cry1 at ZT08 and ZT20. (Bottom tracks) Z-score and signed $-\log _{10}(p)$ show rhythmic contacts between the promoter region and the intronic region. (Black) Cry1 TSS bait $\left(P<10^{-16}\right.$ at peak); (brown) Cry1 intron 1 bait $\left(P<10^{-8}\right.$ at peak). (C) Same as $B$, targeting the Gys2 promoter. (Bottom tracks) Same as $B$ for Gys2 TSS bait $\left(P<10^{-4}\right.$ at peak). (Brown) Gys2 exon8 bait $\left(P<10^{-18}\right.$ at peak). Vertical dotted lines show the positions locally of maximal differential chromatin interactions. 
differential signal covered the entire Cry1 locus, the largest difference was localized-peaking $26 \mathrm{~kb}$ downstream from the TSS in the first Cry1 intron-and highly significant $\left(P<5.5 \times 10^{-17}\right.$ at the peak) (Fig. $1 \mathrm{~B}$, bottom tracks, vertical dotted line at the left). A secondary peak was observed near the $3^{\prime}$ end of the Cry 1 transcript.

To further validate the time-dependent contacts, we placed a bait at the $+26-\mathrm{kb}$ intronic site (reciprocal $4 \mathrm{C}$ seq). The reciprocal $4 \mathrm{C}$-seq confirmed the increased contact frequency with the Cry1 promoter region at ZT20 compared with ZT08 (Fig. 1B bottom tracks, brown solid line; Supplemental Fig. S2A). In fact, the reciprocal differential signal peaked $7 \mathrm{~kb}$ upstream of the Cry1 TSS, a site that was also differentially contacted by the Cry1 TSS bait $\left(P<1.9 \times 10^{-9}\right)($ Fig. 1B, bottom tracks, vertical dotted line at the right; Supplemental Fig. S2A; Supplemental Table S1). Thus, these 4C-seq data in the liver suggested dynamic contacts between the Cry1 promoter and the $+26-\mathrm{kb}$ intronic site as well as the $-7-\mathrm{kb}$ upstream site. Since Cry1 mRNA accumulated rhythmically in the kidney (Supplemental Fig. S3A), we also performed 4C-seq in kidneys. Consistent with the liver data, these sites were also recruited to the Cry1 promoter more frequently at ZT20 than at ZT08 (Supplemental Fig. S3B,C).

Opposite to Cryl, the Gys2 promoter contacted an intragenic region more frequently at ZT08 versus ZT20 (Fig. 1C), with a peak $21 \mathrm{~kb}$ downstream from the TSS in exon $8\left(P<8.7 \times 10^{-5}\right.$ at peak $)$ (Fig. $1 \mathrm{C}$, bottom tracks, black solid line, vertical dotted line at the left), consistent with its anti-phasic rhythmic mRNA accumulation (Supplemental Fig. S1A). This significant differential signal was validated by reciprocal $4 \mathrm{C}$-seq using the exon 8 as bait $\left(P<2.3 \times 10^{-19}\right.$ at peak) (Fig. $1 \mathrm{C}$, bottom tracks, brown solid line, vertical dotted line at the right; Supplemental Fig. S2B). In the kidney, where Gys2 mRNA accumulation was constant and low, this differential signal was absent (Supplemental Fig. S3D-F). Thus, both gene promoters formed DNA loops with neighboring intragenic regions in $c$ is that coincided with the timing of the respective peaks in Cry1 and Gys2 mRNA expression.

\section{The dynamics of chromatin topology depend on BMAL1}

To test whether these dynamic contacts depended on a functional circadian clock, we performed 4C-seq in the livers of clock-deficient animals (Bmal1 knockout) in which Cry1 and Gys2 lost rhythmic expression and were constantly expressed at high and low levels, respectively (Supplemental Fig. S4A,B). In Bmal1 knockout, the Cry1 $+26-\mathrm{kb}$ intronic and $-7-\mathrm{kb}$ upstream regions contacted the promoter at comparable frequencies at ZT20 and ZT08, suggesting static chromatin loops (Fig. 2A,B). For Gys2, the profile between the exon 8 region and the promoter was also static (Fig. 2C,D). Comparing wild-type and Bmal1 knockout at both time points revealed that for Cry1, the loop was locked in a closed conformation (Supplemental Fig. S4C, constitutively high frequencies), and for Gys2, it was locked in an open conformation (Supplemental Fig. S4D, constitutively low frequencies). Thus, the closed and open states of DNA loops concurred with high and low transcription, respectively (Supplemental Fig. S4, cf, A,C and B,D). We note that these 4C profiles suggested a BMAL1-independent interaction upstream of Gys2 (Fig. 2C, lower panels), but this effect was less robust compared with the BMAL1-dependent intragenic looping. As a negative control, we targeted the Hoxd4 locus, which is a transcriptionally silent region in the adult liver. As expected, chromatin contact profiles at the Hoxd4 locus remained static over time in both wild-type and Bmal1 knockout livers (Fig. 2E; Supplemental Fig. S4E). These data thus showed that rhythmic loops in Cry1 and Gys2 depended on the clock TF BMAL1.

\section{Rhythmic DNA loops connect gene promoters with daily active enhancers}

To characterize the interacting genomic regions, we integrated temporal data on DNase-I hypersensitivity sites (DHSs) with ChIP-seq (chromatin immunoprecipitation [ChIP] combined with high-throughput sequencing) data for RNA polymerase II (Pol II), the activity-related chromatin mark H3K27ac (Sobel et al. 2017), and rhythmically active TFs (Rey et al. 2011; Zhang et al. 2015). This allowed us to assess whether the rhythms in DNA contacts coincided with rhythms in activity-related chromatin marks. For Cry1, RNA Pol II and H3K27ac signals peaked near ZT20 (Fig. 3), while, for Gys2, they peaked near ZT08 (Fig. 4). However, while RNA Pol II signals extended throughout the gene bodies, H3K27ac signals were spatially confined around the largest differential contact precisely at sites marked with DHSs. Furthermore, both the 26-kb downstream intronic site and the 7-kb upstream site of the Cry1 TSS contained a RORE-responsive element (RRE) and were bound by the circadian TFs REVERB $\alpha$ and ROR $\gamma$ (Fig. 3; Supplemental Table S4; Zhang et al. 2015). In mouse fibroblasts, the intronic RRE is required for proper timing of Cry1 expression (Ukai-Tadenuma et al. 2011). The interacting Gys2 exon 8 site was bound by the clock regulator BMAL1 at ZT06 (Rey et al. 2011) and by REV-ERBa at ZT10 (Fig. 4). This indicated that DNA contacts connected local rhythmically active enhancer elements with the promoters of Cry1 and Gys2.

\section{Deleting the Cryl intronic enhancer in mice shortens the circadian locomotor period}

To study the function of the rhythmic chromatin interactions, we generated a mouse strain $($ Cry1 $1 \Delta e)$ with a 300base-pair (bp) deletion covering the Cry1 intronic enhancer (Supplemental Fig. S5A,B). We measured spontaneous locomotor activity in constant darkness and observed that Cry1 $\Delta e$ animals had an endogenous circadian period that was significantly shorter $\left(P<1.1 \times 10^{-5}, t\right.$-test $)$ by 15 min compared with wild-type littermates (Fig. 5A; Supplemental Fig. S5C,D). Such period shortening is in the range of classic short period core clock mutants such as Per1 (Cermakian et al. 2001) and Clock (Debruyne et al. 2006). As Cry1 loss of function shortens the circadian period by 1.2-h (van der Horst et al. 1999), our noncoding DNA deletion suggests a Cry1 hypomorph. 
A
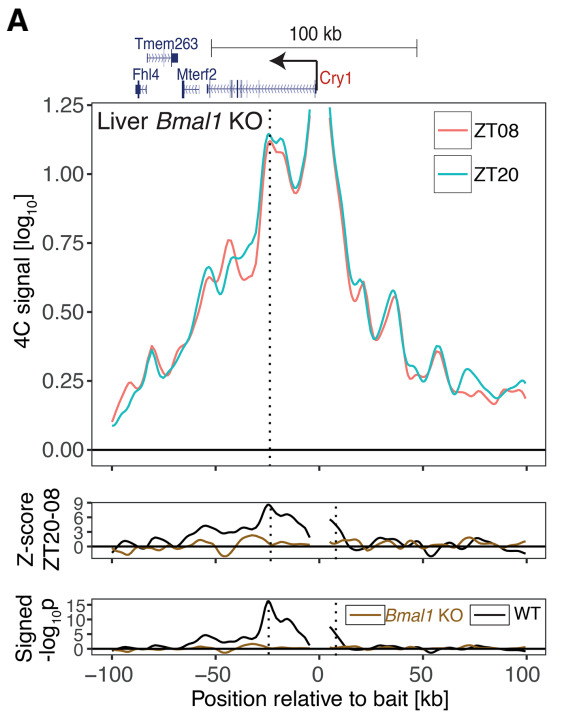

B

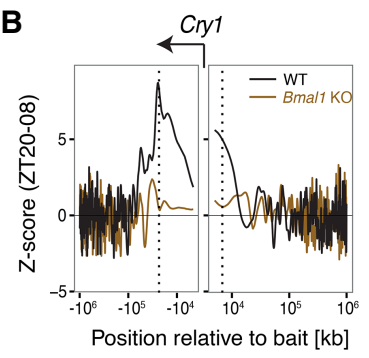

C
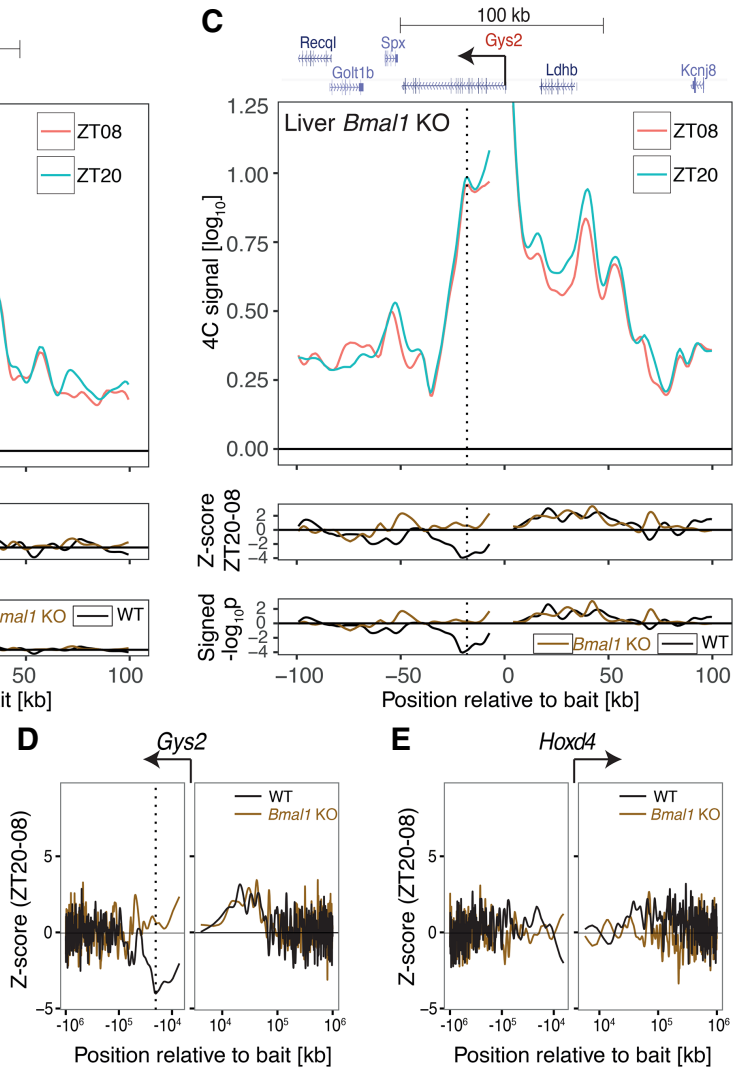

E

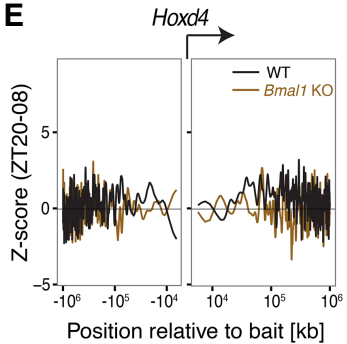

Figure 2. The dynamics of chromatin topology depend on BMAL1. (A, top) 4C-seq signal targeting Cry1 from the livers of Bmal1 knockout mice at ZT20 versus ZT08 shows loss of rhythms in chromatin interactions. (Bottom) Z-score and signed $-\log _{10}(p)$ of differential 4C-seq signal (ZT20-ZT08) in wild-type versus Bmal1 knockout. Vertical lines show BMAL1-dependent rhythmic contacts. (B) Z-score in a 2-Mb genomic region surrounding Cry 1 in wild-type versus Bmal1 knockout. $(C)$ Same as in $A$ but for Gys2 bait. $(D, E)$ Same as in $B$ but for Gys2 $(D)$ and Hoxd4 $(E)$ baits. $B$ and $D$ show that the BMAL1-dependent rhythmic contacts are localized within $100 \mathrm{~kb}$ of the bait.
Expression of Cry1, clock, and clock output genes is perturbed in Cryl $\Delta \mathrm{e}$

To investigate the link between the deletion, promoterenhancer looping, and Cry1 expression in livers and kidneys, we first generated temporal RNA sequencing (RNA-seq) data in Cry1 $\Delta e$ and wild-type littermates under an entraining light-dark cycle. The transcriptomes in Cry1 $\Delta e$ and wild-type littermates were comparable overall in both tissues (Supplemental Fig. S6A). While Cry1 mRNA levels remained rhythmic in both genotypes, likely driven by further regulatory sites (e.g., the TSS and $-7-\mathrm{kb}$ sites), the peak expression at ZT20 was significantly reduced by $27 \%$ in the livers $(15 \%$ in the

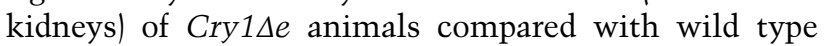
(Supplemental Fig. S6B). Quantifying the intronic reads as a proxy for transcription showed that Cry1 transcription was also phase-advanced in Cry1 $\Delta e$ animals (Supplemental Fig. S6C). Moreover, CRY1 protein abundance in the liver was lower in Cry1 $\Delta e$ compared with wild type, consistent with a reduction in mRNA levels (Supplemental Fig. S6D,E).

As is known in chronobiology, entraining a short period circadian oscillator by an external light-dark cycle leads to a phase advance of internal timing markers (Aschoff and Pohl 1978). This prediction was confirmed in the transcriptome data. Indeed, core clock and clock-controlled genes (Supplemental Table S5) were phase-advanced by, on average, $30 \mathrm{~min}$ in the livers of Cry1 $1 \mathrm{e}$ animals compared with wild type $(P<0.01$ binomial test) (Supplemen- tal Fig. S6F), with Cry1 showing the largest phase advance $(P=0.011$ for livers; $P=0.047$ for kidneys, bootstrap test $)$ (Supplemental Fig. S6B).

\section{The Cry $1 \Delta \mathrm{e}$ mutation disrupts rhythmic chromatin topology}

Next, we explored the dynamics of chromatin topology along the 24-h cycle in liver sampled every $4 \mathrm{~h}$ in wild type and Cry1 $\Delta e$ ( $n=3$ per time point). First, we confirmed oscillatory chromatin interactions in Gys2 in wild type. Indeed, the Gys2 promoter rhythmically recruited the $+21-\mathrm{kb}$ enhancer, peaking near ZT08 in both the TSS bait and exon 8 bait $\left(P<10^{-6}\right.$ at the peak harmonic regression) (Supplemental Fig. S7A-C). As negative control, the Hoxd4 bait measured around the clock did not show oscillatory contacts (Supplemental Fig. S7D). For Cry1 wild type, the frequency of contacts between the promoter and the $+26-\mathrm{kb}$ enhancer significantly oscillated, peaking near ZT20 $\left(P<10^{-8}\right.$ at the peak) (Fig. 5B-D; Supplemental Fig. S8A,B). In contrast, in Cry1 $\Delta$ e, the contact frequencies in this region were lower at all time points compared with wild type, and the oscillation was compromised (Fig. 5B-D; Supplemental Fig. S8A,B). Finally, we also estimated chromatin contacts for a bait placed at the $-7-\mathrm{kb}$ upstream enhancer (Fig. 1B, bottom tracks, vertical dotted line at the right), showing oscillation in contact frequency peaking around ZT20 with the $+26-\mathrm{kb}$ intronic enhancer in wild type but 


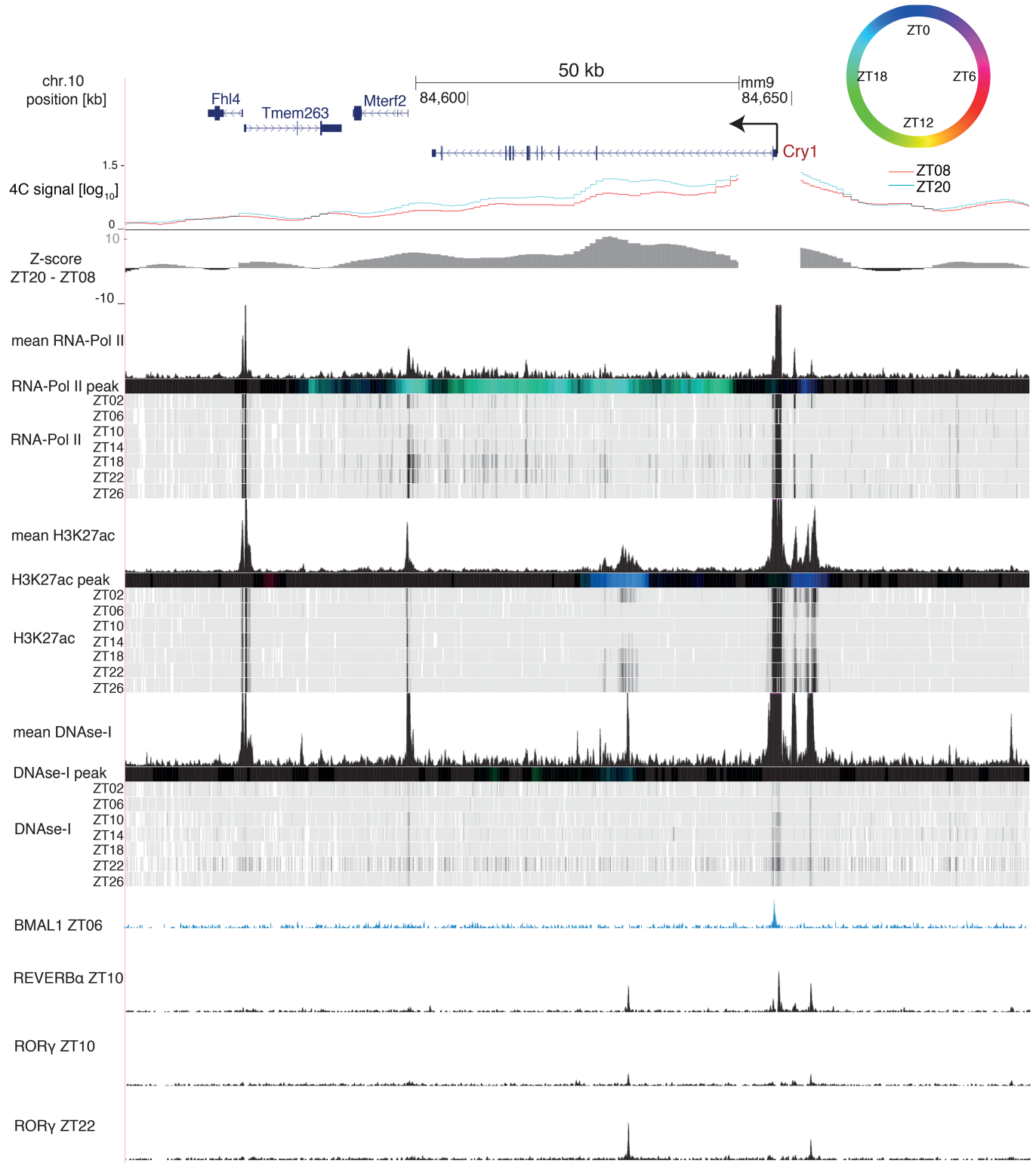

Figure 3. The rhythmic Cry1 loop connects the promoter with a H3K27ac-marked enhancer. The Cry1 genomic region containing 4C-seq signals from Cry1 TSS at ZT08 (red) and ZT20 (blue) and Z-score (ZT20-ZT08) in wild-type livers. RNA Pol II loadings (ChIP-seq), H3K27ac mark (ChIP-seq), and DNase-I signal are from Sobel et al. (2017). Temporally averaged signals and temporal signals of each mark are plotted. Colored bars represent peak times according to the color legend at the top right; black signifies no rhythm (Materials and Methods). BMAL1 ChIP-seq signal is from Rey et al. (2011), and REV-ERBa and ROR $\gamma$ ChIP-seq signals are from Zhang et al. (2015).

nonrhythmic and overall lower contact frequency in Cry1 $\Delta e$ (Supplemental Fig. S8C,D). Decreased contact frequency in Cry1 $\Delta e$ mice indicates that the RRE-containing 300-bp fragment drives the promoter-enhancer loop.

Overall, these data demonstrate robust rhythmic chromatin topology for Cry1 and Gys2, where the frequency of enhancer-promoter contacts is modulated with time of day. Furthermore, deleting a localized noncoding DNA enhancer element (300 bp) in the Cry1 gene could disrupt such rhythms.
The Cryl intronic enhancer modulates transcriptional burst frequency

To analyze whether the Cry1 intronic enhancer modulates transcription, we estimated transcriptional parameters by smRNA-FISH against Cry1 pre-mRNA in the livers of wild-type and Cry1 $\Delta e$ animals at ZT08 and ZT20 (Fig. 6A). Mammalian promoters are irregularly transcribed (transcriptional bursting), as characterized by the burst size and burst frequency (Suter et al. 2011; Bahar Halpern et al. 2015). Taking into account the ploidy of 


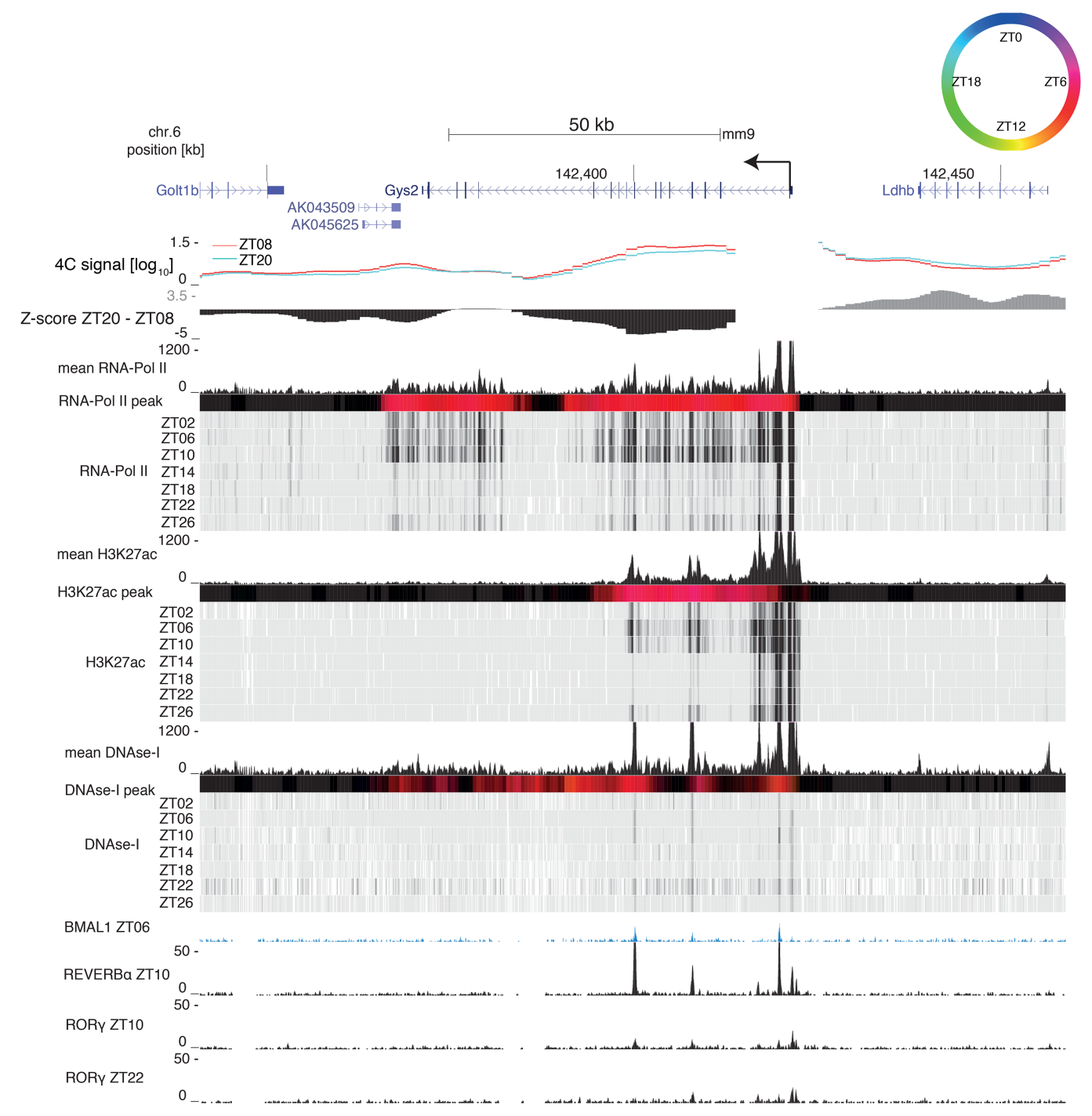

Figure 4. The rhythmic Gys2 loop connects the promoter with a H3K27ac-marked enhancer. The Gys2 genomic region containing 4Cseq signals from the Gys2 TSS at ZT08 (red) and ZT20 (blue) and Z-score (ZT20-ZT08) in wild-type livers. RNA Pol II loadings (ChIP-seq), H3K27ac mark (ChIP-seq), and DNase-I signal are from Sobel et al. (2017). Temporally averaged signals and temporal signals of each mark are plotted. Colored bars represent peak times according to the color legend at the top right; black signifies no rhythm (Materials and Methods). The BMAL1 ChIP-seq signal is from Rey et al. (2011), and the REV-ERBa and ROR $\gamma$ ChIP-seq signals are from Zhang et al. (2015).

liver nuclei (Supplemental Fig. S9A-D), smRNA-FISH showed that Cry1 burst fraction (fraction of active transcription sites in each nucleus, which is proportional to the burst frequency per allele) was 2.2-fold higher at ZT20 compared with ZT08 in wild type (Fig. 6B). Importantly, the burst fraction was reduced by $28 \%$ in Cry $1 \Delta e$ animals at ZT20 (Fig. 6B). In contrast, the burst intensity (proportional to the burst size) was similar in all conditions (Fig. 6C). Thus, the lowered Cry1 mRNA levels in Cry1 10 at ZT20 can be quantitatively explained by the reduced burst fraction. In sum, dynamic enhancer loops modulate transcriptional bursting in mammalian tissues (Bartman et al. 2016; Fukaya et al. 2016); in particular, rhythmic DNA loops involving clock enhancers control burst frequency while maintaining burst size.

\section{Discussion}

In animals, developmental transitions occurring on the time scales of days have been shown to involve remodeled DNA contacts and promoter-enhancer loop formation (Noordermeer et al. 2014). While such dynamics are typically irreversible, we here discovered that chromatin topology in mouse tissues can be locally $(100 \mathrm{~kb}$, in cis) plastic, exhibiting temporal dynamics that are regulated by daily time and the circadian oscillator and thus recur within a 24 -h period. While previous work in cell culture reported dynamic chromatin contacts on larger genomic scales, notably between the Dbp gene and DNA regions on trans chromosomes (Aguilar-Arnal et al. 2013), the genes analyzed here did not show rhythmic chromatin 

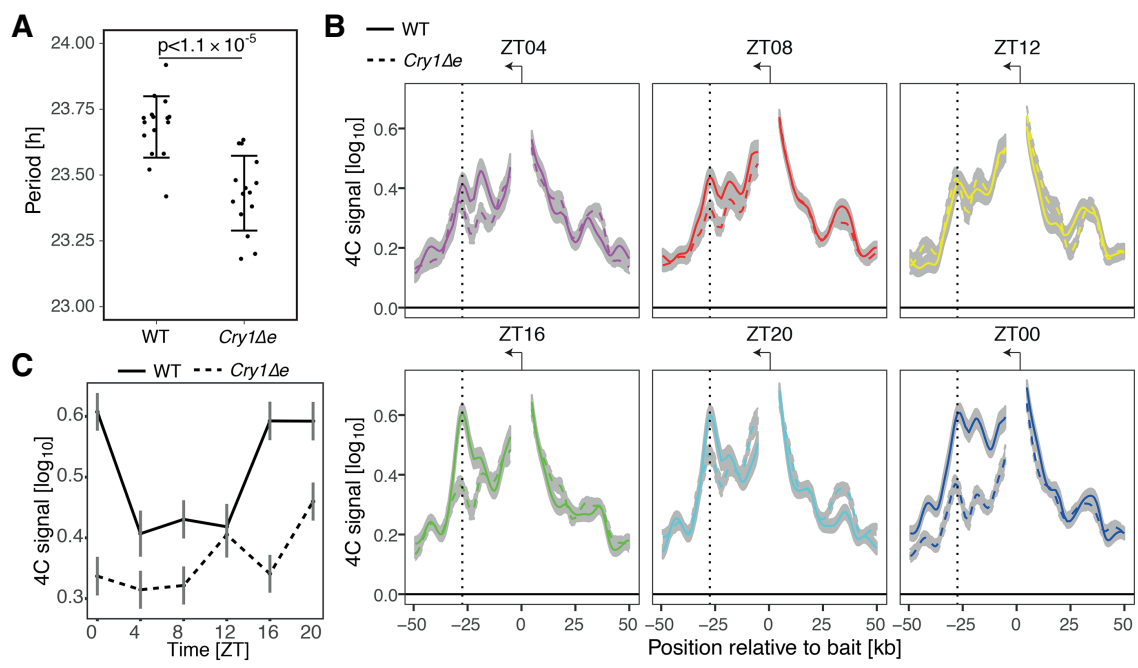

D
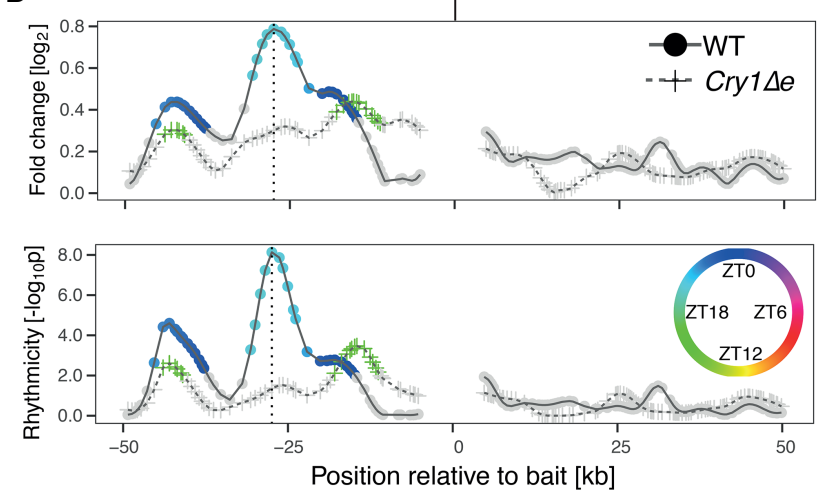

interactions on such scales. We then showed genetically that these rhythmic DNA contacts depend on the clock protein BMAL1 and, in the case of Cry1, a 300-bp intronic RRE-containing enhancer sequence.

How is BMAL1 involved in the formation of these dynamic loops? In the case of Gys2 in Bmal1 knockout mice, the loop is constitutively open, and Gys2 mRNA expression is constitutively low. Combined with the binding of BMAL1 at the looping site, these data strongly argue for a direct involvement of BMAL1. For Cry1, the activator ROR $\gamma$ and the repressor REV-ERBa bind to the Cry 1 intronic enhancer at the expected peak (ZT20) and trough (ZT08) activities, as is typical of functional RREs. We note that while the expression of the RRE-binding repressors ReV-Erba/ $\beta$ is low in Bmal1 knockout, the corresponding activator Rory is constitutively high (Atger et al. 2015). Therefore, the constitutively closed Cry1 loop in the Bmal1 knockout most likely reflects an indirect effect via perturbed REV-ERB and ROR activities. This is further corroborated by the constitutively open state of the Cry 1 promoter-enhancer loop in Cry1 $1 \Delta e$ mice, showing chromatin interactions that are constantly below wild-type trough levels, indicating that loop-promoting factors (for example, RORs) act within the 300-bp element. Therefore, our data suggest a canonical mechanism of enhancer-promoter looping by which sequence-specific TFs
Figure 5. Deleting the Cry1 intronic enhancer in mice shortens the period of the clock and disrupts oscillations in Cry 1 promoter-enhancer contact frequencies. $(A)$ The circadian period of spontaneous locomotor activity is significantly different between Cry1 $1 \Delta e$ and wild-type littermates. The mean period and standard deviation were calculated from 16 wild-type and 15 Cry1 1 e littermates. $P=1.1 \times 10^{-5}, t$-test. (B) 4C-seq signal for Cry1 TSS bait over time in livers (LWMR summarizes $n=3$ animals per group; gray shade shows \pm standard error) in wild-type versus Cry1 $\Delta e$ littermates. Vertical lines show the +26 $\mathrm{kb}$ intronic enhancer. (C) 4C-seq signal over time adjacent to the intronic enhancer. $(D) \log _{2}$ fold change and $-\log _{10}(p)$ from rhythmicity analysis of $4 \mathrm{C}$-seq signal over time. $P<10^{-8}$ at peak, LWMR, $\chi^{2}$ test. Fragments with $P<0.01$ are colored by time of peak contact frequency (color legend is shown at the right). help recruit transcription complexes, which facilitate the function of Pol II at core promoters (Levine and Tjian 2003).

To investigate the effects of the dynamic looping on transcriptional parameters, we complemented bulk 4C-seq and RNA-seq experiments with single-molecule transcript analysis in situ, which revealed that the abol-

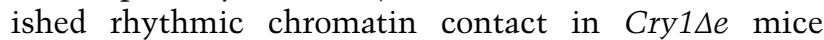
reduced Cry1 transcriptional burst frequency. These results in mammalian tissues contribute to our current understanding of how enhancer loops modulate transcriptional bursting (Bartman et al. 2016; Fukaya et al. 2016). In particular, we showed that rhythmically active clock enhancers can increase burst frequency while not changing burst size.

The ablation of the Cry1 noncoding regulatory element even led to a short period phenotype in locomotor activity. While noncoding genetic variation in humans has been associated recently with circadian clock-related and sleep phenotypes (Allebrandt et al. 2010; Hu et al. 2016), no demonstration of such variation on circadian transcription or behavior has yet been provided. Indeed, previously characterized mutations impacting mammalian circadian behavior have concerned protein-coding regions (Vitaterna et al. 1994; Toh et al. 2001). Here, we provided evidence that noncoding regulatory elements within the 

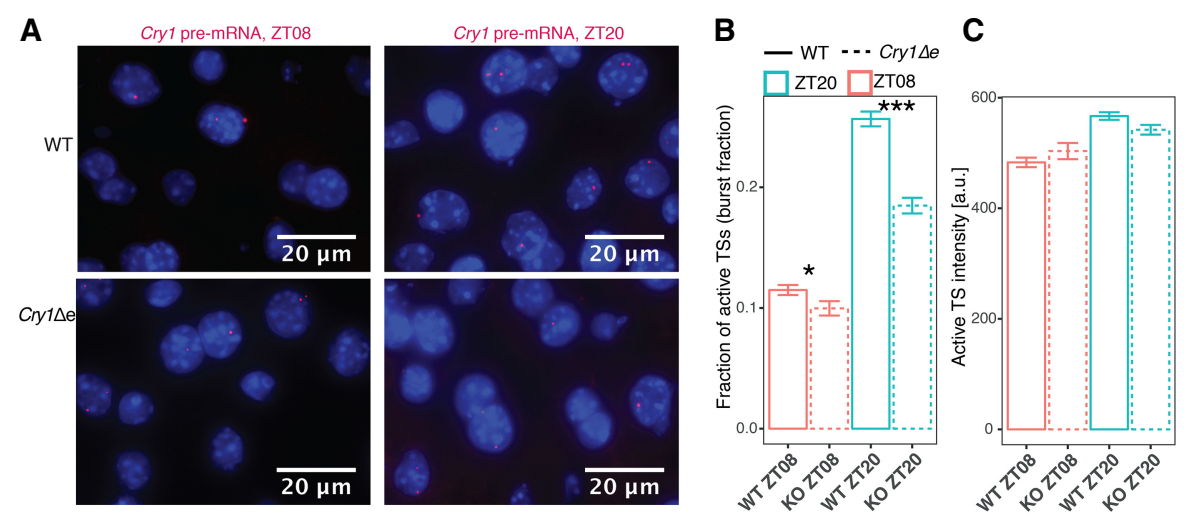

Figure 6. The oscillatory Cry1 promoter-enhancer loop modulates Cry1 transcriptional bursting. (A) smRNA-FISH against Cry1 premRNA in the livers of wild-type (top) and Cry1 $\Delta$ e (bottom) animals at ZT08 (left) and ZT20 (right). Burst fractions (B) and burst intensities $(C)$ measured from images of smRNA-FISH performed against Cry1 pre-mRNA in Cry1 $\Delta e$ (dashed) and wild-type (solid) livers at ZT08 (red) and ZT20 (blue). Burst fraction is the number of active transcription sites in each nucleus divided by the ploidy. $(B, C)$ Shown are the means and standard errors over nuclei collected and pooled from two animals in each of the four conditions (individual animals are analyzed in Supplemental Fig. S9C,D). $n=2191$ wild-type ZT08 nuclei; $n=983$ Cry1 $\Delta e$ ZT08 nuclei; $n=2150$ wild-type ZT20 nuclei; $n=1473$ Cry1 $\Delta e$ ZT20 nuclei. In $\left.B,\left({ }^{*}\right) P<0.05 ;{ }^{* * *}\right) P<0.001, t$-test. In $C$, differences between genotypes are not significant.

core circadian regulatory network can drive dynamic promoter-enhancer looping, modulate temporal transcription, and regulate circadian locomotor behavior.

\section{Materials and methods}

\section{Animal and ethics statement}

All animal care and handling were performed according to Canton de Vaud laws for animal protection (authorization VD2801 [Frédéric Gachon] and VD3109 [Félix Naef]). All experiments were performed on males between 8 and 10 wk old. Bmal1 knockout animals were described previously in Jouffe et al. (2013).

\section{Mouse genome editing by direct knockout using CRISPR-Cas9}

Px-330 plasmids targeting upstream of and downstream from the Cry1 intron1 regulatory region were injected into pronuclei and then transplanted into B6D2F1 pseudopregnant mice at the Ecole Polytechnique Fédérale de Lausanne (EPFL) Transgenic Core Facility (http://tcf.epfl.ch). Pups from the first generation (F0) were then screened for the deletion using the PCR primers indicated in Supplemental Table S2. F0 animals of interest were backcrossed on C57/BL6J wild-type mice, and F1 animals were screened for transmission of the mutation. Heterozygous animals were crossed together to obtain all genotypes of interest. The Ethical Committee of the State of Vaud Veterinary Office, Switzerland, approved all experiments.

\section{Nucleus purification and fixation}

Immediately after sacrifice, $5 \mathrm{~mL}$ of $1 \times$ PBS was perfused through the spleen to flush blood from the liver. Livers and kidneys from individual animals were homogenized and fixed in $4 \mathrm{~mL}$ of $1 \times$ PBS, including $1.5 \%$ formaldehyde, for $10 \mathrm{~min}$ at room temperature. The cross-linking reaction was stopped by adding $25 \mathrm{~mL}$ of ice-cold stop reaction buffer ( $2.2 \mathrm{M}$ sucrose, $150 \mathrm{mM}$ glycine, 10 $\mathrm{mM}$ HEPES at $\mathrm{pH} 7.6,15 \mathrm{mM} \mathrm{KCl}, 2 \mathrm{mM}$ EDTA, $0.15 \mathrm{mM}$ spermine, $0.5 \mathrm{mM}$ spermidine, $0.5 \mathrm{mMDTT}, 0.5 \mathrm{mM}$ PMSF) to the homogenates and was kept for 5 min on ice. Homogenates were then loaded on top of $10 \mathrm{~mL}$ of cushion buffer (2.05 M sucrose, $10 \%$ glycerol, $125 \mathrm{mM}$ glycine, $10 \mathrm{mM}$ HEPES at $\mathrm{pH} 7.6,15 \mathrm{mM} \mathrm{KCl}$, $2 \mathrm{mM}$ EDTA, $0.15 \mathrm{mM}$ spermine, $0.5 \mathrm{mM}$ spermidine, $0.5 \mathrm{mM}$ DTT, $0.5 \mathrm{mM}$ PMSF) and centrifuged at $10^{5} \mathrm{~g}$ for $45 \mathrm{~min}$ at $4^{\circ} \mathrm{C}$. Nuclei were washed twice in $1 \times$ PBS and immediately frozen.

$4 C-\operatorname{seq}$

$4 C$ template preparation $4 \mathrm{C}$ templates were prepared as in Gheldof et al. (2012). Nuclei were resuspended in $1 \mathrm{~mL}$ of a buffer containing $10 \mathrm{mM}$ Tris-HCL (pH 8.0), $10 \mathrm{mM} \mathrm{NaCl}, 0.2 \%$ NP-40, and $1 \times$ protease inhibitor cocktail (Complete Mini EDTA-free protease inhibitor cocktail; Sigma-Aldrich); kept for $15 \mathrm{~min}$ on ice; and washed twice with $1 \times$ DpnII buffer (New England Biolabs). Thirty million nuclei were resuspended in $1 \times$ DpnII buffer (New England Biolabs) containing 0.1\% SDS and incubated for 10 min at $65^{\circ} \mathrm{C}$. Triton X-100 was added to $1 \%$ final concentration. Chromatin was digested overnight with $400 \mathrm{U}$ of DpnII (New England Biolabs) at $37^{\circ} \mathrm{C}$ with shaking. After heat inactivation, digestion efficiency was evaluated by both DNA visualization on agarose gels and quantitative PCR using primer pairs covering multiple restriction sites. Chromatin was then ligated with $3000 \mathrm{U}$ of T4 DNA ligase (New England Biolabs) in an 8$\mathrm{mL}$ final volume for $4 \mathrm{~h}$ at $16^{\circ} \mathrm{C}$ plus $1 \mathrm{~h}$ at room temperature. The cross-linking reaction was reverted by the addition of $50 \mu \mathrm{L}$ of $10 \mathrm{mg} / \mathrm{mL}$ proteinase $\mathrm{K}$ and incubation overnight at $65^{\circ} \mathrm{C}$. DNA was purified by multiple phenol/chloroform extractions, resuspended in TE buffer $(\mathrm{pH} 8.0)$ containing RNase A, and incubated for $30 \mathrm{~min}$ at $37^{\circ} \mathrm{C}$. Ligation efficiency was evaluated by loading DNA on an agarose gel. Libraries were digested with $1 \mathrm{U}$ of NlaIII per microgram of template (New England Biolabs) overnight at $37^{\circ} \mathrm{C}$, and digestion was controlled by visualization on an agarose gel. After heat inactivation, digested products were ligated with $2000 \mathrm{U}$ of T4 DNA ligase (New England Biolabs) for $4 \mathrm{~h}$ at $16^{\circ} \mathrm{C}$ in a $14-\mathrm{mL}$ final volume. Circularized products were purified and resuspended in TE buffer ( $\mathrm{pH}$ 8.0). 4C templates were prepared in four biological replicates in wildtype mouse livers and kidneys and three biological replicates in the livers of Bmal1 knockout and Cry1 $\Delta e$ and wild-type littermates (Supplemental Table S1). 
Inverse PCR and sequencing in wild-type and Bmal1 knockout mouse livers and kidneys Six-hundred nanograms of $4 \mathrm{C}$ template was used for PCR amplification using Sigma-Aldrich long-template PCR system with bait-specific inverse primers conjugated to Illumina sequencing adaptors (primer sequences are in Supplemental Table S3) in a final volume of $50 \mu \mathrm{L}$ in the following PCR program: $2 \mathrm{~min}$ at $94^{\circ} \mathrm{C}$ followed by 30 cycles of $15 \mathrm{sec}$ at $94^{\circ} \mathrm{C}, 1 \mathrm{~min}$ at $55^{\circ} \mathrm{C}$, and $3 \mathrm{~min}$ at $68^{\circ} \mathrm{C}$ and a final extension of $7 \mathrm{~min}$ at $68^{\circ} \mathrm{C}$. PCR were performed in parallel reactions with $6 \times 100 \mathrm{ng}$ of template for each sample. PCR products were purified with the AMPure XP beads system (Beckman Coulter), and amplification profiles were analyzed by fragment analyzer and then sequenced on Illumina HiSeq 2000 machines using single-end 100-bp read length.

Inverse PCR and sequencing in the livers of Cry1 $1 \Delta$ e and wild-type littermates Six-hundred nanograms of $4 \mathrm{C}$ template was used for PCR amplification using Sigma-Aldrich long-template PCR system with two-step PCR system from Illumina. Bait-specific inverse primers conjugated to Illumina sequencing adaptors (primer sequences are in the Supplemental Table S3) were used in a first PCR reaction in a final volume of $50 \mu \mathrm{L}$ with the following program: $2 \mathrm{~min}$ at $94^{\circ} \mathrm{C}$ followed by 20 cycles of $15 \mathrm{sec}$ at $94^{\circ} \mathrm{C}, 1$ min at $55^{\circ} \mathrm{C}$, and $3 \mathrm{~min}$ at $68^{\circ} \mathrm{C}$ and a final extension of $7 \mathrm{~min}$ at $68^{\circ} \mathrm{C}$. PCRs were performed in parallel reactions with $6 \times 100$ ng of template for each sample. PCR products were purified with the AMPure XP beads system (Beckman Coulter). Purified products were pooled and used as the template of a second PCR reaction with Nextera XT index kit version2 primers (FC-1312004 ) in a final volume of $50 \mu \mathrm{L}$ with the following program: 2 min at $94^{\circ} \mathrm{C}$ followed by 10 cycles of $15 \mathrm{sec}$ at $94^{\circ} \mathrm{C}, 1 \mathrm{~min}$ at $55^{\circ} \mathrm{C}$, and $3 \mathrm{~min}$ at $68^{\circ} \mathrm{C}$ and a final extension of $7 \mathrm{~min}$ at $68^{\circ} \mathrm{C}$. PCR products were purified with the AMPure XP beads system (Beckman Coulter) and then sequenced on NextSeq 500 machines using single-end 75-bp read length.

\section{C-seq analysis}

Preprocessing computational methods Demultiplexed Fastq files were mapped to the mouse genome $(\mathrm{mm} 9)$ using Bowtie 2 with default HTSstation parameters (http://htsstation.epfl.ch). Since each restriction fragment contained two mapping sites (two ends of the fragment), the fragment score was computed as the average of the number of reads per mapping site.

Quality control of 4 C-seq data Samples with $\geq 75 \%$ of restriction fragments without any counts in a window of $\pm 1 \mathrm{Mb}$ upstream of and downstream from each bait were not analyzed (Supplemental Table S1). The first five fragments upstream of and downstream from the bait (10 total) were not considered in the analysis because they mostly contained partially digested and self-ligated products.

Normalization and LWMR We follow a method developed recently in Yeung et al. (2018) with minor modifications. Briefly, raw read counts for each sample were library size-rescaled by the normalized sum of the read counts on the cis chromosome (excluding 10 restriction fragments around the bait). To control the variability of low signals, in subsequent analyses, the fragment counts $c$ in each sample were log transformed using the variable

$$
Y=\log _{10}\left(\frac{c}{P}+1\right)
$$

with $P=500$. A weighted linear model was then fit locally using a Gaussian window $\left(\sigma_{G}=2500 \mathrm{bp}\right)$ centered on the fragment of interest. For each position, nearby $4 \mathrm{C}$-seq signals $(Y)$ were modeled with fragment effects $a_{i}$ and condition effects $b_{j}$ (which can be time, tissue, or genotype). In LWMR, these parameters were estimated by minimizing the weighted sum $S$ of squared residuals across replicates $r: S=\operatorname{argmin}_{a, b} \Sigma_{i, j, r} \mathrm{~W}_{i, j}\left(Y_{i, j, r}-a_{i}-b_{j}\right)^{2}$, with weights $W_{i, j}$ defined as $W_{i, j}=W_{g, i} \times W_{s, j}$, where $W_{g, i}$ is the Gaussian smoothing kernel at position $i$, and $w_{s, j}$ is a condition weight based on the number of samples with nonzero counts on fragment $i$. Specifically, we used $w_{s}=0.5,1.5,2.5,3.5$, or 4.5 for fragments with zero, one, two, three, or four replicates showing nonzero counts, which down-weighs positions with high dropout rates. To estimate the statistical significance for differential contacts (for example, ZT20 vs. ZT08), we propagated the estimated uncertainty (standard errors for locally weighted regression) in the corresponding $b$ values to calculate $Z$-scores and used regularized $t$ statistics with $n-p$ degrees of freedom (DOF; $n$ is the number of data points within window, and $p$ is the number of parameters). For the analysis of 24-h rhythmicity in contacts (weighted harmonic regression), we proceeded analogously by propagating the uncertainty in the $b$ s for the six time points to that in the squared 24-h Fourier coefficient and used the $\chi^{2}$ test with two DOF (owing to the real and imaginary parts). For each set of samples, we computed the regularized residual variance as

$$
\tilde{\sigma}^{2}=\hat{\sigma}^{2}+\sigma_{\min }^{2} \exp \left(-\frac{\bar{b}}{b_{s}}\right),
$$

with $\hat{\sigma}^{2}$ as the estimator of the squared residuals, $\bar{b}$ as the estimated signal across samples, and $b_{s}=\log _{10}(2) . \sigma_{\min }^{2}$ prevents artificially small variance from positions of high dropout rates and is estimated from the distribution of $\tilde{\sigma}^{2}$ across all fragments. $\sigma_{\text {min }}$ ranges from 0.06 to 0.16 (same units as $Y$ ), depending on the bait (Supplemental Table S1).

\section{H3K27ac and RNA Pol II ChIP-seq and DNase-I-seq analysis}

Bam files from GSE60578 (Sobel et al. 2017) were analyzed in genomic regions $\pm 1 \mathrm{Mb}$ from the $4 \mathrm{C}$-seq baits. There, read counts were binned in 500-bp intervals and normalized by the library size. The amplitude and phase of the $\log _{2}$ read counts of each of the three signals were calculated for each bin after applying a running average of seven bins (three bins upstream, three bins downstream, and one bin in the center) to smooth the signal. Obtained rhythmic amplitudes and phases were compared with differential 4C-seq signals. The rhythmic signal in each bin [phase, amplitude, and $\left.-\log _{10}(p)\right]$ was mapped to a color using the hue, saturation, and value (HSV) color scheme. Hue $h$ was defined by the phase of the oscillation, with blue as ZT0. The saturation $s$ was set to 1 . The value $V$ was set to a color if both amplitude $X_{a}$ and $-\log _{10}(p) X_{p}$ were beyond thresholds $k_{a}=1, k_{p}=4.5$; otherwise, the color was set to black. To obtain smooth transitions, $V$ was calculated using a Hill function with Hill coefficient $n=5$ and

$$
V=\min _{i \in(a, p)}\left(\frac{-\log \left(x_{i}\right)^{5}}{k_{i}^{5}-\log \left(x_{i}\right)^{5}}\right) .
$$

For TF-binding site predictions (Supplemental Table S4), we used weight matrices of TFs defined by SwissRegulon (Pachkov et al. 2007; http://swissregulon.unibas.ch/fcgi/sr/downloads).

\section{RNA-seq in the livers and kidneys of Cryl $1 \mathrm{e}$ and wild-type littermates}

Parts of the livers and kidneys from the animals used for temporal 4C-seq experiments were frozen in liquid nitrogen immediately after sacrifice. Organs were homogenized in $4 \mathrm{M}$ guanidine thiocyanate, $25 \mathrm{mM}$ sodium citrate, $1 \% \beta$-mercaptoethanol, and 0.2 
M sodium acetate. Nucleic acids were extracted with phenol: chloroform:isoamylalcohol, and RNA was precipitated with 4 $\mathrm{M} \mathrm{LiCl}$. RNA concentration and purity were measured using nanodrop, and the quality was controlled by fragment analyzer. Poly-A-selected RNA was sequenced on NextSeq 500 machines using single-end 75-bp read length. mRNA levels were quantified using kallisto version 0.42.4 (mm10) (Bray et al. 2016).

RNA-seq in the livers and kidneys of Bmall knockout and wild-type mice

To complement the mouse liver wild-type and Bmal1 knockout RNA-seq data (GSE73554), transcriptomes of kidneys from wild-type animals were measured following the same protocol as in Atger et al. (2015). mRNA levels were quantified using the same method as in Atger et al. (2015).

Circadian period estimation in Cryl $\Delta \mathrm{e}$ animals and wild-type littermates

Estimation of the circadian period was performed as in Diessler et al. (2017). Briefly, 8- to 10-wk-old males were single-caged and kept under $12 \mathrm{~h} / 12 \mathrm{~h}$ light/dark cycle for $14 \mathrm{~d}$ and switched to constant darkness for $21 \mathrm{~d}$. During the $5 \mathrm{wk}$ of the experiment, the locomotor activity was recorded with passive infrared sensors. Data were sampled with 5-min resolution and analyzed using the $\chi^{2}$ periodogram function in the ClockLab software (ActiMetrics). Food and water were available ad libitum during the entire experiment.

\section{Western blotting}

Liver cytoplasmic extracts were prepared as described previously (Jouffe et al. 2013). Protein extract concentrations were quantified using a BCA protein assay kit (Thermo Fisher Scientific), and 20 $\mu \mathrm{g}$ of liver protein extract was resolved by SDS-PAGE using standard procedures. Densitometry analyses of the blots were performed using the ImageJ software. Naphtol blue and black staining of the membranes was used as a loading control and served as a reference for normalization of the quantified values. CRY1 antibody (1/500) was from Abcam (ab104736).

\section{smRNA-FISH on mouse liver sections}

Parts of the livers from the same animals used in the 4C-seq and RNA-seq were collected, immediately embedded in O.C.T. compound (Tissue-Tek, Sakura-Finetek USA), and snap-frozen. The RNA-FISH was done on 8- $\mu$ m cryosections using a RNAscope probe for Cry1 pre-mRNA (Cry1_intron1, catalog no. 500231) according to the manufacturer's instructions for the RNAscope fluorescent multiplex assay (Advanced Cell Diagnostics). Nuclei were counterstained with DAPI, and sections were mounted with ProLong Gold anti-fade mountant (Molecular Probes).

Microscope image acquisition, quantification, and ploidy assignment

The sections were imaged using a Leica DM5500 wide-field microscope equipped with a CCD camera (DFC 3000) for fluorescence (Leica Microsystem) and a motorized stage. $Z$-stacks were aquired $(0.2 \mu \mathrm{m}$ between each $Z$ position, 40 images per frame) with an oil immersion $63 \times$ objective. The images were quantified using ImageJ. To detect the fluorescent RNA-FISH spots, a Laplacian filter was applied on a maximal projection, and local maxima were computed. Transcription site fluorescent intenstities (burst size) were quantified on the sum projection of the nine best-focused stacks per image. Total transcription site signals were com- puted using a mask of $3 \times 3$ pixels. Nuclei were detected using filters, thresholding, and watershed transformation. Ploidy $(2 \mathrm{~N}$, $4 \mathrm{~N}$, or $8 \mathrm{~N}$ ) was assigned to the nuclei based on their diameter (Bahar Halpern et al. 2015). A four-component Gaussian mixture model was fitted to the diameter distribution (package "mixtools" in R). Nuclei with a probability of $>0.7$ to belong to one of the three inferred populations with the smallest means were assigned to $2 \mathrm{~N}, 4 \mathrm{~N}$, and $8 \mathrm{~N}$, respectively. The Gaussian distribution with the largest variance captured outliers in nucleus diameters $(>15-18 \mu \mathrm{m})$ and were discarded. Burst fraction was calculated as the number of active transcription sites in each nucleus divided by its estimated ploidy, and these fractions were then averaged over the entire populations of nuclei (Fig. 5B,C). For Supplemental Figure S10C, we modeled the number of active transcription sites with genotype-dependent slopes and compared it with a reduced model without a genotype effect (lme4 function in R, likelihood ratio test). For Supplemental Figure S10D, we modeled the mean intensity of intronic dots with genotype-dependent intercepts and compared it with a reduced model with a single intercept.

\section{Data availability}

Raw and processed sequencing data generated from this study (4C-seq and RNA-seq) have been submitted to Gene Expression Omnibus under accession number GSE101423.

\section{Acknowledgments}

We thank Isabelle Barde at the École Polytechnique Fédérale de Lausanne (EPFL) Transgenic Core Facility for help generating the Cry1 1 e mouse strain, Jessica Dessimoz at the EPFL Histology Core Facility for smRNA-FISH experiments, Jacques Rougemont and Marion Leuleu for help with analysis of 4C-seq, Keith Harshman and Corinne Peter from the Genomic Technologies Facility at University of Lausanne, and Bastien Mangeat at the EPFL Gene Expression Core Facility for 4C-seq and RNA-seq. This work was supported by Swiss National Science Foundation grant 31003A153340 (to F.N.), European Research Council grant ERC-2010StG-260667 (to F.N.), and École Polytechnique Fédérale de Lausanne. J.Y. benefits from the Natural Sciences and Engineering Research Council of Canada Post-graduate Studies Doctoral scholarship. Computations and analyses were performed at Vital-IT (http://www.vital-it.ch).

Author contributions: J.M., J.Y., and F.N. conceived the study; J.Y., K.G., C.G., and F.N. performed the formal analyses; J.M., C.H., D.M., C.J., D.N., and Y.E. performed the investigations; J.M., J.Y., and F.N. wrote the manuscript; J.M., J.Y., P.F., F.G., and F.N. reviewed and edited the manuscript; P.F., F.G., and F.N. supervised the study; and P.F., F.G., and F.N. acquired the funding.

\section{Note added in proof}

While this manuscript was in review, similar 24-h rhythmic promoter-enhancer chromatin interactions at the Cry1 locus were reported (Kim et al. 2018).

\section{References}

Aguilar-Arnal L, Hakim O, Patel VR, Baldi P, Hager GL, SassoneCorsi P. 2013. Cycles in spatial and temporal chromosomal 
organization driven by the circadian clock. Nat Struct Mol Biol 20: 1206-1213.

Allebrandt KV, Teder-Laving M, Akyol M, Pichler I, Muller-Myhsok B, Pramstaller P, Merrow M, Meitinger T, Metspalu A, Roenneberg T. 2010. CLOCK gene variants associate with sleep duration in two independent populations. Biol Psychiatry 67: 1040-1047.

Aschoff J, Pohl H. 1978. Phase relations between a circadianrhythm and its Zeitgeber within range of entrainment. Naturwissenschaften 65: 80-84.

Atger F, Gobet C, Marquis J, Martin E, Wang J, Weger B, Lefebvre G, Descombes P, Naef F, Gachon F. 2015. Circadian and feeding rhythms differentially affect rhythmic mRNA transcription and translation in mouse liver. Proc Natl Acad Sci 112: E6579-E6588.

Bahar Halpern K, Tanami S, Landen S, Chapal M, Szlak L, Hutzler A, Nizhberg A, Itzkovitz S. 2015. Bursty gene expression in the intact mammalian liver. Mol Cell 58: 147-156.

Bartman CR, Hsu SC, Hsiung CCS, Raj A, Blobel GA. 2016. Enhancer regulation of transcriptional bursting parameters revealed by forced chromatin looping. Mol Cell 62: 237-247.

Bass J, Lazar MA. 2016. Circadian time signatures of fitness and disease. Science 354: 994-999.

Bray NL, Pimentel H, Melsted P, Pachter L. 2016. Near-optimal probabilistic RNA-seq quantification. Nat Biotechnol 34: 525-527.

Cermakian N, Monaco L, Pando MP, Dierich A, Sassone-Corsi P. 2001. Altered behavioral rhythms and clock gene expression in mice with a targeted mutation in the Periodl gene. EMBO J 20: 3967-3974.

Debruyne JP, Noton E, Lambert CM, Maywood ES, Weaver DR, Reppert SM. 2006. A clock shock: mouse CLOCK is not required for circadian oscillator function. Neuron 50: 465-477.

Dekker J, Marti-Renom MA, Mirny LA. 2013. Exploring the three-dimensional organization of genomes: interpreting chromatin interaction data. Nat Rev Genet 14: 390-403.

Diessler S, Kostic C, Arsenijevic Y, Kawasaki A, Franken P. 2017. Rail frees mice from the repression of active wake behaviors by light. eLife 6: e23292.

Doi R, Oishi K, Ishida N. 2010. CLOCK regulates circadian rhythms of hepatic glycogen synthesis through transcriptional activation of Gys2. J Biol Chem 285: 22114-22121.

Fukaya T, Lim B, Levine M. 2016. Enhancer control of transcriptional bursting. Cell 166: 358-368.

Fulco CP, Munschauer M, Anyoha R, Munson G, Grossman SR, Perez EM, Kane M, Cleary B, Lander ES, Engreitz JM. 2016. Systematic mapping of functional enhancer-promoter connections with CRISPR interference. Science 354: 769-773.

Ghavi-Helm Y, Klein FA, Pakozdi T, Ciglar L, Noordermeer D, Huber W, Furlong EE. 2014. Enhancer loops appear stable during development and are associated with paused polymerase. Nature 512: 96-100.

Gheldof N, Leleu M, Noordermeer D, Rougemont J, Reymond A. 2012. Detecting long-range chromatin interactions using the chromosome conformation capture sequencing (4C-seq) method. Methods Mol Biol 786: 211-225.

Griffin EA Jr, Staknis D, Weitz CJ. 1999. Light-independent role of CRY1 and CRY2 in the mammalian circadian clock. Science 286: 768-771.

Hu Y, Shmygelska A, Tran D, Eriksson N, Tung JY, Hinds DA. 2016. GWAS of 89,283 individuals identifies genetic variants associated with self-reporting of being a morning person. Nat Commun 7: 10448.

Irimia JM, Meyer CM, Peper CL, Zhai L, Bock CB, Previs SF, McGuinness OP, DePaoli-Roach A, Roach PJ. 2010. Impaired glucose tolerance and predisposition to the fasted state in liver glycogen synthase knock-out mice. I Biol Chem 285: 12851-12861.

Jouffe C, Cretenet G, Symul L, Martin E, Atger F, Naef F, Gachon F. 2013. The circadian clock coordinates ribosome biogenesis. PLOS Biol 11: e1001455.

Kim YH, Marhon SA, Zhang Y, Steger DJ, Won KJ, Lazar MA. 2018. Rev-erba dynamically modulates chromatin looping to control circadian gene transcription. Science doi: 10.1126/science.aao6891.

Kuznetsova T, Wang SY, Rao NA, Mandoli A, Martens JH, Rother N, Aartse A, Groh L, Janssen-Megens EM, Li G, et al. 2015. Glucocorticoid receptor and nuclear factor $\mathrm{\kappa B}$ affect three-dimensional chromatin organization. Genome Biol 16: 264.

Levine M, Tjian R. 2003. Transcription regulation and animal diversity. Nature 424: 147-151.

Mermet J, Yeung J, Naef F. 2017. Systems chronobiology: global analysis of gene regulation in a 24-hour periodic world. Cold Spring Harb Perspect Biol 9: a028720.

Noordermeer D, Leleu M, Schorderet P, Joye E, Chabaud F, Duboule D. 2014. Temporal dynamics and developmental memory of 3D chromatin architecture at Hox gene loci. eLife 3: $\mathrm{e} 02557$.

Pachkov M, Erb I, Molina N, van Nimwegen E. 2007. SwissRegulon: a database of genome-wide annotations of regulatory sites. Nucleic Acids Res 35: D127-D131.

Palstra RJ, Tolhuis B, Splinter E, Nijmeijer R, Grosveld F, de Laat W. 2003. The $\beta$-globin nuclear compartment in development and erythroid differentiation. Nat Genet 35: 190-194.

Rey G, Cesbron F, Rougemont J, Reinke H, Brunner M, Naef F. 2011. Genome-wide and phase-specific DNA-binding rhythms of BMAL1 control circadian output functions in mouse liver. PLoS Biol 9: e1000595.

Sanborn AL, Rao SS, Huang SC, Durand NC, Huntley MH, Jewett AI, Bochkov ID, Chinnappan D, Cutkosky A, Li J, et al. 2015. Chromatin extrusion explains key features of loop and domain formation in wild-type and engineered genomes. Proc Nat1 Acad Sci 112: E6456-E6465.

Sanyal A, Lajoie BR, Jain G, Dekker J. 2012. The long-range interaction landscape of gene promoters. Nature 489: 109-113.

Schibler U, Gotic I, Saini C, Gos P, Curie T, Emmenegger Y, Sinturel F, Gosselin P, Gerber A, Fleury-Olela F, et al. 2015. Clock-talk: interactions between central and peripheral circadian oscillators in mammals. Cold Spring Harb Symp Quant Biol 80: 223-232.

Sobel JA, Krier I, Andersin T, Raghav S, Canella D, Gilardi F, Kalantzi AS, Rey G, Weger B, Gachon F, et al. 2017. Transcriptional regulatory logic of the diurnal cycle in the mouse liver. PLoS Biol 15: e2001069.

Suter DM, Molina N, Gatfield D, Schneider K, Schibler U, Naef F. 2011. Mammalian genes are transcribed with widely different bursting kinetics. Science 332: 472-474.

Takahashi JS. 2017. Transcriptional architecture of the mammalian circadian clock. Nat Rev Genet 18: 164-179.

Toh KL, Jones CR, He Y, Eide EJ, Hinz WA, Virshup DM, Ptacek LJ, Fu YH. 2001. An hPer2 phosphorylation site mutation in familial advanced sleep phase syndrome. Science 291: 1040-1043.

Ukai-Tadenuma M, Yamada RG, Xu H, Ripperger JA, Liu AC, Ueda HR. 2011. Delay in feedback repression by cryptochrome 1 is required for circadian clock function. Cell 144: 268-281.

van der Horst GT, Muijtjens M, Kobayashi K, Takano R, Kanno S, Takao M, de Wit J, Verkerk A, Eker AP, van Leenen D, et al. 
1999. Mammalian Cry1 and Cry2 are essential for maintenance of circadian rhythms. Nature 398: 627-630.

Vitaterna MH, King DP, Chang AM, Kornhauser JM, Lowrey PL, McDonald JD, Dove WF, Pinto LH, Turek FW, Takahashi JS. 1994. Mutagenesis and mapping of a mouse gene, Clock, essential for circadian behavior. Science 264: 719-725.

Xu Y, Guo W, Li P, Zhang Y, Zhao M, Fan Z, Zhao Z, Yan J. 2016. Long-range chromosome interactions mediated by cohesin shape circadian gene expression. PLoS Genet 12: e1005992.

Yeung J, Mermet J, Jouffe C, Marquis J, Charpagne A, Gachon F, Naef F. 2018. Transcription factor activity rhythms and tissue-specific chromatin interactions explain circadian gene expression across organs. Genome Res 28: 182-191.
Zhang R, Lahens NF, Ballance HI, Hughes ME, Hogenesch JB. 2014. A circadian gene expression atlas in mammals: implications for biology and medicine. Proc Natl Acad Sci 111: 16219-16224.

Zhang Y, Fang B, Emmett MJ, Damle M, Sun Z, Feng D, Armour SM, Remsberg JR, Jager J, Soccio RE, et al. 2015. Discrete functions of nuclear receptor Rev-erba couple metabolism to the clock. Science 348: 1488-1492.

Zhao H, Sifakis EG, Sumida N, Millan-Arino L, Scholz BA, Svensson JP, Chen X, Ronnegren AL, Mallet de Lima CD, Varnoosfaderani FS, et al. 2015. PARP1- and CTCF-mediated interactions between active and repressed chromatin at the lamina promote oscillating transcription. Mol Cell 59: 984-997. 


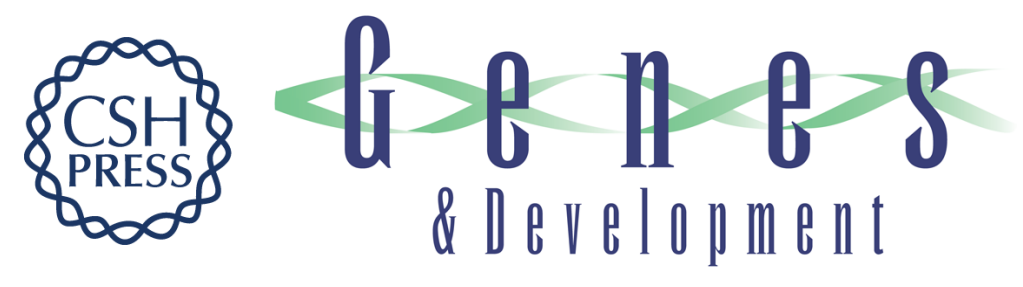

\section{Clock-dependent chromatin topology modulates circadian transcription and behavior}

Jérôme Mermet, Jake Yeung, Clémence Hurni, et al.

Genes Dev. 2018, 32: originally published online March 23, 2018

Access the most recent version at doi:10.1101/gad.312397.118

\section{Supplemental http://genesdev.cshlp.org/content/suppl/2018/03/23/gad.312397.118.DC1 \\ Material}

Related Content

References

Creative

Commons

License

Email Alerting

Service
A day in the life of chromatin: how enhancerpromoter loops shape daily behavior Benjamin J. Weidemann, Kathryn Moynihan Ramsey and Joseph Bass

Genes Dev. March , 2018 32: 321-323

This article cites 44 articles, 17 of which can be accessed free at: http://genesdev.cshlp.org/content/32/5-6/347.full.html\#ref-list-1

Articles cited in:

http://genesdev.cshlp.org/content/32/5-6/347.full.html\#related-urls

This article, published in Genes \& Development, is available under a Creative Commons License (Attribution 4.0 International), as described at

http://creativecommons.org/licenses/by/4.0/.

Receive free email alerts when new articles cite this article - sign up in the box at the top right corner of the article or click here.

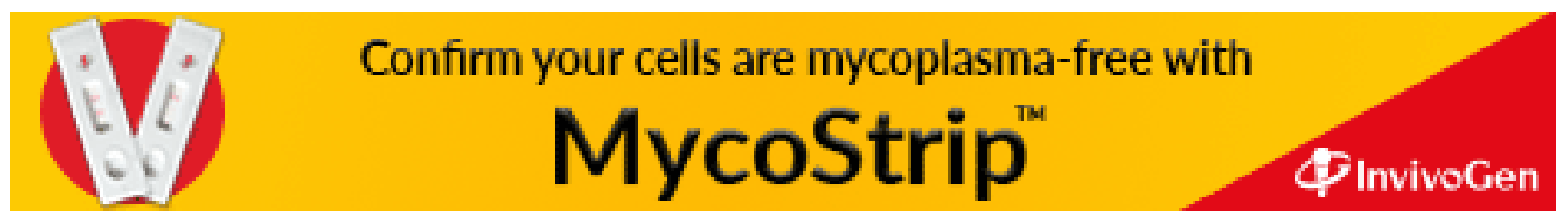

